of calorimetric methods has taken in the remark able progress made in the past three decades. Prof. Onsager gave the principal lecture of the technical sessions on "Co-operative Phenomena", a field in which he has developed much of the basic theory. Many papers at each Calorimetry Conference describe experimental studies of co-operative phenomena, and Onsager outlined the approaches one may take in seeking a theoretical understanding of such effects. Admitting that three-dimensional treatments of critical phenomena by statistical mechanics seem hopelessly complex, he dwelt mostly on more simplified treatments that give results.

In addition to the objective of promoting better calorimetric research, the Conference also is concerned with publication policies relating to calorimetric and thermodynamic articles. A "Resolution regarding Published Calorimetric Data" adopted by the eighth Conference in 1953 has proved to be valuable to editors and authors alike in establishing consistent policies based on the opinions of experts in the field. Because calorimetric research has expanded into many areas not covered by the 1953 resolution, the fourteenth Conference established a committee headed by J. P. McCullough to consider revising and extend. ing the earlier recommendations. Drs. Edgar F.
Westrum, jun. (University of Michigan), and Stig Sunner (University of Lund, Sweden) presented a proposal of the Commission on Thermodynamics of the International Union of Pure and Applied Chemistry for a joint meeting in 1961 of the Calorimetry Conference and the Subcommissions on Experimental Thermochemistry and Experimental Thermodynamics. The Conference unanimously approved the proposal for a joint meeting to be held either before or after the biennial meeting of the Union that year in Montreal, Canada. Plans will begin immediately for what should be one of the most important eonferences ever held in the field of calorimetry.

At the annual election the following members were appointed to Conference offices: Chairman, Dr. J. P. McCullough (Petroleum Thermodynamics Laboratory, Bureau of Mines); Chairman-Elect, Dr. D. W. Osborne (Argonne National Laboratory); Directors, 1959-62, Dr. N. E. Phillips (University of California, Berkeley) and Dx. J. M. Sturtevant (Yale University). Other officers include: SecretaryTreasurer, Dr. C. E. Messer (Tufts University) and Directors, Dr. David White, Dr. D. H. Andrews (Johns Hopkins University), Dr. J. E. Kunzler (Bell Telephone Laboratories), and Dr. J. A. Morrison (National Research Council, Ottawa).

\title{
SECOND AUSTRALIAN SPECTROSCOPY CONFERENCE
}

T HE second Australian Spectroscopy Conference, convened by Dr. A. L. G. Rees (Division of Chemical Physics, Commonwealth Scientific and Industrial Research Organization) and held in the Chemistry Department of the University of Melbourne during June 1-3, was opened by Prof. J. S. Anderson, who welcomed the 110 participants and the four exhibitors of commercial spectroscopic equipment. The first session of the conference was devoted to ultra-violet spectra and began with a review by Prof. N. S. Bayliss (Chemistry Department, University of Western Australia) of recent theoretical work on solvent effects. He directed attention to the calculations of Polansky on the interaction between two $\mathrm{H}$ atoms which predict a red shift in the atomic spectrum beyond a critical distance and a blue shift at closer distances, to the calculations by Longuet-Higgins and Pople of the red shift in the spectra of non-polar solutes in non-polar solvents arising from dispersive forces, and to McRee's formulations of the case of polar solute and polar solvent. The McRae formula predicts a frequency shift between absorption and fluorescence arising from the change of dipole moment between the ground and excited states, thus providing a method for measuring the dipole moments of excited states for comparison with calculated values.

One set of contributed papers in this section dealt with the spectra of aromatic hydrocarbons. Drs. G. R. Hunt and I. G. Ross (Physical Chemistry Department, University of Sydney) discussed a vibrational analysis of the $7000 \mathrm{~A}$. and $3500 \mathrm{~A}$. absorption systems of azulene which appears to confirm the predictions of Pariser and of Moffitt concerning the nature of the excited states. Dr. L. E. Lyons and Mr. G. C. Morris (Physical Chemistry Department, University of Sydney) presented results on the absorption of anthracene vapour $(38,000$ to $\left.60,000 \mathrm{~cm}^{-1}\right)$. They confirmed the second $\pi-\pi$ transition as allowed and also observed four members of a Rydberg series converging to an ionization potential of $6.81 \mathrm{eV}$. Dr. N. S. Ham (Division of Chemical Physics, Commonwealth Scientific and Industrial Research Organization) reported some calculations by the free-electron model, with electronic interaction, of the spectrum of the perinaphthenylium cation $\mathrm{C}_{13} \mathrm{H}_{9}{ }^{+}$, which agree well with the reported spectrum and also predict an unreported absorption band at about $600 \mu$.

Studies by Dr. I. G. Ross and E. J. Wells (Physical Chemistry Dopartment, University of Sydney) on the interesting tetrahedral molecules $\mathrm{OsO}_{4}$ and $\mathrm{RuO}_{4}$ failed to reproduce the extensive fine structure reported in the room temperature spectra by Langseth and Qviller in 1934. The authors gave a vibrational analysis of their spectra and used the energy-lovel scheme of Ballhausen and Liehr to assign the two allowed transitions. A theoretical paper by Dr. E. G. McRae (Division of Chemical Physics, Commonwealth Scientific and Industrial Research Organization) was concerned with electronically excited states of aggregated identical molecules; the intra-molecular vibrations were explicitly included. Two limiting cases were recognized, depending on whether the interaction onergy was large or small with respect to the vibrational energy. An interpretation of the $J$ band of N,N'-diethyl-pseudo-cyanine was offered on the basis of this theory.

Dr. H. A. McKenzie (Division of Food Preservation and Transport, Commonwealth Scientific and Industrial Research Organization) spolse on the difference spectra in acid solutions and in urea solutions of bovine serum albumin, ovalbumin and conalbumin.

Dr. L. E. Lyons (Physical Chemistry Department, University of Sydney), in opening the session on solid state spectroscopy, described a number of the effects 
observed in ionic solids and semi-conductors. Such effects include structure in the absorption edge of the conduction band, hydrogen-like absorption series due to excitons, the high intensity of the conduction band absorption of germanium due to the low effective mass of the electrons, intervalence band transitions in germanium, and the magnetic splitting of the conduction-levels in InSb. The use of cyclotron resonance in determining the presence of excitons and in evaluating effective masses was also noted. Crystal field effects in the spectra of inorganic complexes and the Davydov splitting in molecular crystals were also discussed.

Dr. J. A. Friend (Chemistry Department, University of Tasmania) and Dr. Lyons have identified two transitions in the crystal spectrum of sodium nitrate, an allowed one at $2000 \mathrm{~A}$. and a weak $n-\pi$ transition at 2870 A. Dr. J. Ferguson (Division of Chemical Physics, Commonwealth Scientific and Industrial Research Organization) has analysed the polarized spectra of microcrystals of some cobalt (II) tetrahedral and octahedral complexes. The absorption spectra of the tetrahedral complexes of the type $\mathrm{CoPy}_{2} \mathrm{X}_{2}$ are modified by intermolecular interaction in the crystal while the octahedral complexes can be interpreted by simple crystal field theory. Mr. J. E. A. Alderson (Physics Department, University of Western Australia) discussed the luminescence spectra of thallium-doped potassium iodide in the spectrum range $600 \mathrm{~A}$. to $2500 \mathrm{~A}$. recorded on a normal incidence grating spectrometer with a photomultiplier as detector. Dr. L. E. Lyons, Dr. J. R. Walsh and Mr. J. W. White (Physical Chemistry Department, University of Sydney) presented the polarized visible spectrum of single crystals of phthalocyanine. An attempt was made to calculate the crystal spectrum using the Davydov theory, but the error in estimating the dipole vector is too large to allow an unambiguous assignment of the crystal levels.

Two papers on vacuum spectroscopic technique were presented. One by Mr. R. S. Crisp (Physics Department, University of Western Australia) described a grazing incidence, photon-counting grating spectrometer, working in the range 40-1000 A. It has been found that the soft X-ray band emission spectra obtained with this instrument change with the material of the grating (aluminium to glass), with time, and with the order in which the grating is used. These effects have no explanation at the moment. J. V. Sullivan (Division of Chemical Physics, Commonwealth Scientific and Industrial Research Organization) described a double-beam photoelectric $1 \mathrm{~m}$. grating spectrometer, which has been tested down to $1500 \mathrm{~A}$. The monochromator employs a concave grating in a new type of mounting in which deviations from the Rowland circle lead to no detectable loss in resolution. The double-beam system involves grazing incidence reflexion to split the exit beam and two electronically coupled photo-multipliers to record the spectra. Examples of spectra recorded over the range $1500-6000 \mathrm{~A}$. were presented.

The second day of the conference began with a review of some of the infra-red, Raman and microwave work of 1958 by Prof. A. N. Hambly (Canberra University College). Topics discussed included : accurate determination of molecular geometries from gaseous Raman and microwave spectra ; information on torsional modes barrier heights, barrier tunnelling and rotational isomerism from spectra in the cæsium iodide and microwave regions; pressure-induced transitions in gases. Perturbation effects and solvent effects need further study, for the reviewer pointed out that despite considerable work on the subject, the intensities of infra-red bands in polar solvents still do not accord with the theories.

In a research paper Prof. Hambly and J. G. Allpress (Chemistry Department, University of Melbourne) showed that infra-red spectra can be used to follow some solid state reactions. They illustrated their remarks by showing spectra of the products of reaction between $\mathrm{U}_{3} \mathrm{O}_{8}$ and alkali or alkaline-earth halides. They could detect differences that were not discernible in X-ray powder patterns.

Dr. E. Spinner (Australian National University. Canberra) discussed the vibration spectra and structure of the hydrochlorides of urea, thiourea and acetamide. Infra-red and Raman spectra suggest that the cations are formed by the addition of a proton to the nitrogen rather than to the oxygen atom and that the $\mathrm{C}-\mathrm{N}$ bond in an amide is a pure single bond. Drs. N. S. Ham and J. B. Willis (Division of Chemical Physics, Commonwealth Scientific and Industrial Research Organization) dealt with the infra-red and Raman spectra of some isothiocyanates. They have identified the symmetrical NCS stretching frequency in - $\mathrm{CH}_{2} \mathrm{NCS}$ types at about $670 \mathrm{~cm} .^{-1}$, and at about $930 \mathrm{~cm} .^{-1}$ in aromatic - NCS compounds. They also gave a detailed interpretation of the complex structure of the strong NCS characteristic band near $2100 \mathrm{~cm}^{-1}$. A.J. Costoulas (Department of Chemistry, University of New South Wales) gave a complete vibrational assignment for methyl-isothiocyanate. He agreed with Ham and Willis in the reassignment of the band at $676 \mathrm{~cm}^{-1}$ to the symmetrical NCS stretching frequency.

Prof, A. N. Hambly and R. H. Laby (Chemistry Department, University of Melbourne) discussed some criteria for allocating displacements of $X-\mathrm{H}$ bond stretching frequencies to the formation of hydrogen bonds. These are a double absorption band for weak intermolecular H-bonds and most weak intramolecular H-bonds, a double absorption band at low concentration in non-polar solvents for stronger intermolecular H-bonds, and a large displacement of frequency but no doubling on dilution for strong intramolecular $\mathrm{H}$-bonds. Some exceptions were illustrated by the spectra of some ortho-amino acetophenones and anthranilic esters. The stable conformations of a series of methyl-cyclo-hexanols were reported by Dr. A. R. H. Cole and G. T. A. Müller (Chemistry Department, University of Western Australia). Small but significant differences in the stretching frequencies of axial (3627-32 cm.-1) and equatorial $\left(3622 \cdot 5-23 \mathrm{~cm}^{-1}\right)$ hydroxyl groups were used to determine the conformations. The methyl group was found to have a greater tendency to be equatorial than the hydroxyl group. A. G. Moritz (Department of Organic Chemistry, University of Adelaide) has examined the overall appearance of the $3000 \mathrm{~cm}^{-1}$ region in the infra-red spectra of a number of methyl-substituted polycyclic hydrocarbons. He has confirmed the general applicability of the correlation between the pattern of this region and the free valence number of the position of substitution in the parent hydrocarbon, a correlation first observed by Fuson and Josien for the methyl-1:2-benzanthracenes.

The results of a normal co-ordinate analysis of the 33 planar vibrations of naphthalene were presented by D. E. Freeman and Dr. I. G. Ross (Physical 
Chemistry Department, University of Sydney). An iterative procedure of adjusting the force constants was used to force agreement with seven of the frequencies the assignment of which is most certain. This necessitates some reassignments, and the resultant interpretation is in general agreement with the work of Luther.

The simplest molecule discussed at the conference was hydrogen deuteride the weak dipole vibration spectrum of which was the subject of a paper by Dr. R. A. Durie (Coal Research Station, Commonwealth Scientific and Industrial Research Organization). The internuclear distance and the anharmonicity constants were calculated from measurements of the rotational structure of the $(1,0),(2,0)$, and $(3,0)$ vibration bands. Dr. D. W. Posener (Division of Electrotechnology, Commonwealth Scientific and Industrial Research Organization) presented the only paper on microwave spectroscopy. He has measured the electric field gradients at the oxygen and hydrogen nuclei in $\mathrm{HDO}$ and $\mathrm{D}_{2} \mathrm{O}$ and has begun a measurement of the magnetic hyperfine splittings.

The following papers which were generally concerned with spectrographic apparatus and methods were also presented. Dr. H. Gollnow (Mt. Stromlo Observatory, Canberra) described a precision photoelectric setting device (accuracy about $0.5 \mu$ ) for the measurement of diffuse spectral lines. A fast-scanning infra-red $(1-5 \mu)$ spectrometer equipped with a cooled lead-tellurium detector was described by Dr. J. Tregallas-Williams (Defence Standards Laboratories. Maribyrnong). Another paper from the same laboratories, by G. L. White, discussed the application of polynomials fitted by 'least squares' methods, instead of the Friedel-McKinney equation, for the calibration of infra-red spectrometers. Some molecular spectra excited by shock waves were shown by Dr. C. L. Cook (Weapons Research Establishment, Salisbury), and the part played by a photographic spectrograph in routine control in the steel industry was outlined by J. H. Savage (Australian Iron and Steel, Port Kembla). Another paper on emission spectroscopy was given by J. M. Nobbs (Defence Standards Laboratories, South Australia), who described some of the effects of pulse shape on the spectral characteristics of pulsed discharges. $\mathrm{H}_{\Theta}$ pointed out that the exponential shapes commonly used yielded lower sensitivities than most other shapes.

The final morning session of the conference took the form of a small symposium on atomic absorption spectroscopy, with a review and five research papers on the subject. A. Walsh (Division of Chemical Physics, Commonwealth Scientific and Industrial Research Organization) reviewed the present status of emission and atomic absorption spectroscopy. Recent advances in emission spectroscopy include better sources, the use of photomultipliers and the availability of vacuum instruments. The use by Margoshes and Scribner of a plasma-jet as a spectroscopic source was a new development which will be followed with great interest. However, the need for standards remains. This results essentially from three types of interference, namely, chemical, radiative and excitative. Atomic absorption spectroscopy avoids two of these but not the chemical interferences. In the opinion of the reviewer the value of atomic absorption spectroscopy has been established and it seems that any analysis that can be done by flame emission methods using atomie lines ean be done as well and often better by atomic absorption methods.

J. E. Allan (Department of Agriculture, Harnilton, New Zealand) described the analysis of magnesium by atomic absorption. The measurement is carried out simultaneously with flame emission analyses for calcium, sodium and potassium, the other major cations present in plants and soils. The magnesium determination can be performed with the same ease as, and probably with greater reliability than, calcium, sodium and potassium determinations. The minor elements zine, iron and manganese have also been determined by atomic absorption directly on plant ash solutions or soil extracts, but copper at present requires prior extraction with an organic solvent. D. J. David (Division of Plant Industry, Commonwealth Scientific and Industrial Research Organization) spoke about some of the chemical interferences encountered in applying atomic absorption to the analysis of plant material and soil extracts. In plant material analyses, he finds no interference for zinc, iron, copper and magnesium but he finds that calcium absorption is depressed by phosphate, aluminium and silicate. This interference is eliminated with 0.6 per cent magnesium ions and 2 per cent, volume/ volume, sulphuric acid. With soil extracts the atomic absorption analysis is straightforward for sodium, potassium and magnesium, but in this case the interference with calcium is controlled by 0.15 per cent strontium ions. These two papers showed that many analyses important in plant and animal nutrition can be carried out successfully by the atomic absorption method.

Dr. J. B. Willis (Division of Chemical Physics, Commonwealth Scientifie and Industrial Research Organization) discussed the determination of blood serum calcium and magnesium levels by atomic absorption. Large quantities of strontium ions or the disodium salt of ethylene diamine tetracetic acid are needed to overcome the interference of phosphate and protein. Duplicate measurements for calcium and magnesium can be made directly on about $0.25 \mathrm{ml}$. of serum diluted 10-20 fold. Dr. P. Brownwell (Botany Department, University of Adelaide) is interested in analysing for very small quantities (about 0.01 p.p.m.) of sodium, which is a micronutrient for Atriplex vesicaria. The flame photometrie results are misleading when the calcium concentration exceeds $0.02 M$. The atomic absorption method using 'Si-ro-spec', a simple instrument designed by Box and Walsh for this purpose, at present enables accurate measurements of about 1 p.p.m. sodiun in the presence of large excesses of calcium $(4 M)$ and potassium.

The final contribution, by Dr. B. M. Gatehouse and Mr. A. Walsh (Division of Chemical Physies, Commonwealth Scientific and Industrial Research Organization), was concerned with the direct analysis of metals and alloys. By sputtering from a metal surface in the presence of about $1 \mathrm{~mm}$. mercury pressure of neon they were able to obtain a calibration curve and to determine 1 p.p.m. silver in a copper-silver alloy. They suggested that this method may be suitable for elements such as aluminium and boron which do not give atoms in the flame.

In a concluding discussion members agreed unanimously that a standing committeo should be set up to organize similar conferences at regular intervals.
NoRMAN S. HAM 